$\begin{array}{cc}\text { ACADEMIA ROMÂNĂ } & \text { Rev. Roum. Chim., } \\ \text { Revo, 65(5), 433-445 } \\ \text { Revue Roumaine de Chimie } & \text { DOI: } 10.33224 / \text { rrch.2020.65.5.03 } \\ \text { http://web.icf.ro/rrch/ } & \end{array}$

\title{
ECCENTRICITY BASED TOPOLOGICAL INDICES OF CHAIN OCTAHEDRON STRUCTURE
}

\author{
Dan GUO, ${ }^{\mathrm{a},{ }^{*}}$ Donggang TAO,${ }^{\mathrm{b}}$ Muhammad Kamran SIDDIQUI,,${ }^{\mathrm{c},}$ Muhammad NAEEM, ${ }^{\mathrm{d}}$ \\ Abdul Qudair BAIG ${ }^{\mathrm{d}}$ and Muhammad Razwan AZHAR ${ }^{\mathrm{d}}$ \\ ${ }^{\mathrm{a} C}$ College of Computer, Tonghua Normal University, tonghua, 134000, China \\ ${ }^{\mathrm{b}}$ Tonghua Educational Bureautonghua, 134000, China \\ ${ }^{c}$ Department of Mathematics, COMSAT University Islamabad, Lahore Campus, 54000, Pakistan \\ ${ }^{\mathrm{d} D e p a r t m e n t}$ of Mathematics and Statistics, Institute of Southern Punjab, Multan, 66000, Pakistan
}

Graph theory has much advancement in the field of mathematical chemistry. Nowadays, chemical graph theory has become very popular among researchers because of its wide applications in mathematical chemistry. The molecular topological descriptors are the numerical in- variants of a molecular graph and are very useful for predicting their bioactivity.

A great variety of such indices are studied and used

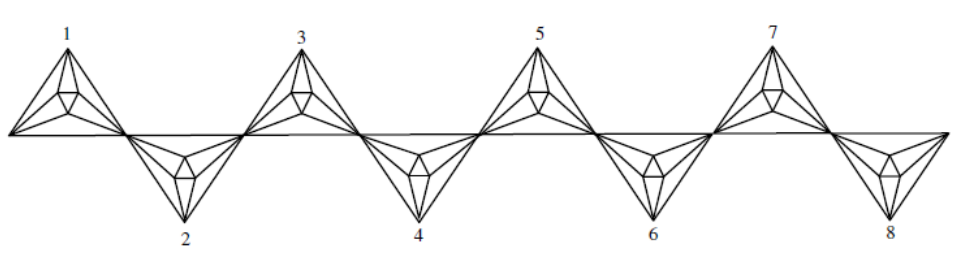
in theoretical chemistry, pharmaceutical researchers, in drugs and in different other fields. In this article, we study the chemical graph of Chain Octahedron structure and compute the Total Eccentricity, Average Eccentricity, Atom bond connectivity index and Geometric arithmetic index of Chain Silicate. Furthermore, we give analytically closed formulas of these indices which are helpful in studying the underlying topologies.

\section{INTRODUCTION}

Cheminformatics is new subject which is a combination of chemistry, mathematics and information science. There is a considerable usage of graph theory in chemistry. Chemical graph theory is the topology branch of mathematical chemistry which implements graph theory to mathematical modeling of chemical occurrence. There is lot of research which is done in this area in the last few decades see details. ${ }^{1-5}$ This theory contributes a major role in the field of chemical sciences. Some references are given, which hopefully demonstrate the importance of this field. ${ }^{6,7,10-12,14,17-19}$ Let $\mathrm{G}=(\mathrm{V}$, E) be a graph, where $\mathrm{V}$ is a non-empty set of vertices and $\mathrm{E}$ is a set of edges. The chemical graph theory applies graph theory to mathematical modeling of molecular phenomena, which is helpful for the study of molecular structure. This theory contributes a prominent role in the field of chemical sciences. Chemical compounds have a variety of applications in chemical graph theory, drug design, etc. The manipulation and examination of chemical structural information is made conceivable by using molecular descriptors. A great variety of topological indices are studied and used in theoretical chemistry, pharmaceutical researchers. In chemical graph theory, there are many topological indices for a connected graph, which are helpful in study of chemical molecules. Development of chemical science had an important effect by this theory.

If $p, q \in V(G)$, then the distance $d(p, q)$ between $\mathrm{p}$ and $\mathrm{q}$ is defined as the length of any shortest path in $\mathrm{G}$ connecting $\mathrm{p}$ and $\mathrm{q}$. Eccentricity

\footnotetext{
*Corresponding authors: guodan_1978@163.com or kamransiddiqui75@gmail.com
} 
is the distance of vertex $u$ from the farthest vertex in G. In mathematical form,

$$
\varepsilon(u)=\max \{d(u, v), \forall v \in V(G)\}
$$

The eccentric connectivity polynomial of a graph $\mathrm{G}$ is defined ${ }^{1,2}$ As we know that the first derivative of ECP $(\mathrm{G}, \mathrm{x})$ evaluated at $\mathrm{x}=1$ is called the eccentric connectivity index.

On the other hand, the eccentric connectivity index of the molecular graph $\mathrm{G}$ in 1997 is introduced by: ${ }^{22}$

$$
E C P(G, x)=\sum_{v \in V} d_{v} \times x^{\epsilon(v)} .
$$

where $\varepsilon(\mathrm{v})$ is the maximum distance between $\mathrm{v}$ and any other vertex $u$ of $G$. The Total-eccentricity index is introduced by Farooq et al., ${ }^{4}$ which is defined as,

$$
\xi(G)=\sum_{v \in V} d_{v} \times \varepsilon(v)
$$

where $\varepsilon(v)$ represents eccentricity of vertex . The Total-eccentricity index is introduced by Farooq et al., ${ }^{4}$ which is defined as,

$$
\zeta(G)=\sum_{v \in V} \varepsilon(v)
$$

The average eccentricity avec $(\mathrm{G})$ of a graph $\mathrm{G}$ is the mean value of eccentricities of all vertices of a graph, that is,

$$
\operatorname{avec}(G)=\frac{1}{n} \sum \varepsilon_{\mathrm{i}}
$$

The eccentricity based geometric-arithmetic index of a graph $\mathrm{G}$ is defined as, ${ }^{8}$

$$
G A_{4}=\sum_{u v \in E(G)} \frac{2 \sqrt{\varepsilon(u) \times \varepsilon(v)}}{\varepsilon(u)+\varepsilon(v)}
$$

A new version of $A B C$ index is introduced by Farahani ${ }^{20}$ which is defined as,

$$
A B C_{\mathrm{s}}=\sum_{u v E E(G)} \sqrt{\frac{\varepsilon(u)+\varepsilon(v)-2}{\varepsilon(u) \times \varepsilon(v)}}
$$

Recently the eccentric atom-bond connectivity index of linear polycene parallelogram benzenoid is intro. The average eccentricity and standard deviation for all Sierpiński graphs $\mathrm{Sn}$ is established by. ${ }^{16}$ The extremal properties of the average eccentricity, conjectures and AutoGraphiX, about the average eccen- tricity are obtained by ${ }^{13}$ The bounds on the mean eccentricity of graph and also the change in mean eccentricity when a graph is replaced by a subgraph is established by. ${ }^{3}$ For trees with fixed diameter, fixed matching number and fixed number of pendent vertices, the lower and upper bounds of average eccentricity are found by. ${ }^{23-33}$

\section{RESULTS AND DISCUSSION}

In this section, we discuss the chain Octahedron structure and give close formulae of certain topological indices for this network. Here we find the analytically closed results of eccentric connectivity polynomial, eccentric connectivity index, total eccentricity index, average eccentricity index, eccentricity based geo- metric arithmetic and atom bond connectivity indices for chain Octahedron structure.

An octahedron graph shown in Fig. 1, is a polyhedral graph corresponding to the skeleton of a Platonic solid. This Platonic graph consists of 6 vertices and 12 edges. The analogs of this structure play a vital role in the fields of reticular chemistry, which deals with the synthesis and properties of metal-organic frameworks.$^{15-35}$

The different types of octahedral structures arise from the ways these octahedra can be connected. A chain octahedral structure of dimension $\mathrm{n}$ denoted as $\mathrm{C} \mathrm{H}$ On is obtained by arranging $\mathrm{n}$ octahedra lin- early as shown in Fig. 2. The number of vertices and edges of $\mathrm{C} \mathrm{H}$ On are $5 n+1$ and $12 n$ respectively. We compute the exact formulas for the above mentioned topological indices of chain octahedral structure as follows.

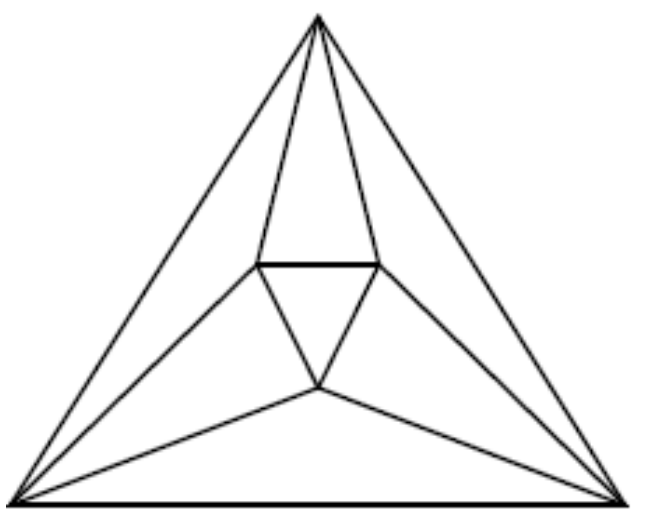

Fig. 1 - Structure of an Octahedron. 


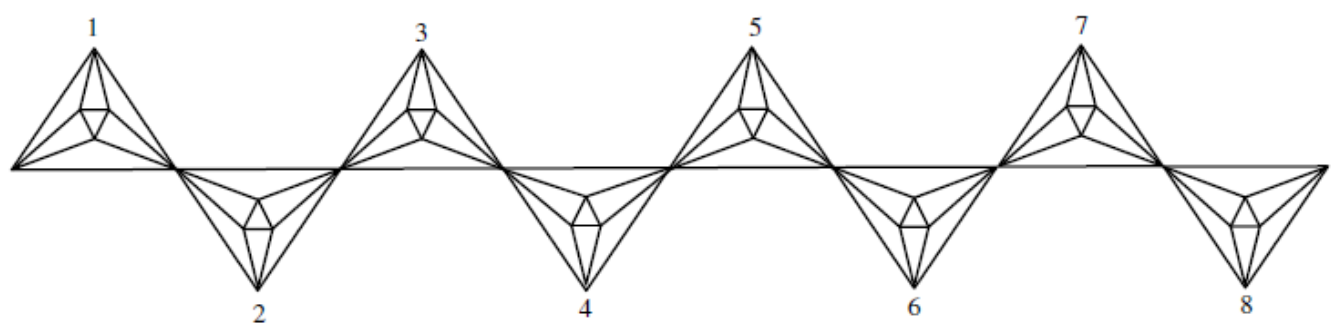

Fig. 2 - Chain Octahedral structure C HO8.

\section{Table 1}

Vertex partition of Chain Octahedron structure for (n-levels) where $\mathrm{n}$ is odd, based on degree and eccentricity of each vertex with existence of their frequencies

\begin{tabular}{c|c|c|l}
\hline $\mathbf{d}(\mathbf{u})$ & $\boldsymbol{\varepsilon ( u )}$ & frequency & Range of k and n \\
\hline 4 & $\mathrm{k}$ & 2 & $k=\frac{n+3}{2}, n \geq 3$ \\
\hline 4 & $\mathrm{k}$ & 8 & $\frac{n+5}{2} \leq k \leq n, n \geq 5$ \\
\hline 4 & $\mathrm{k}$ & 10 & $k=n+1, n \geq 3$ \\
\hline 4 & $\mathrm{k}$ & 2 & $k=n+2, n \geq 3$ \\
\hline 8 & $\mathrm{k}$ & 2 & $\frac{n+3}{2} \leq k \leq n, n \geq 3$ \\
\hline
\end{tabular}

\section{Table 2}

Vertex partition of Chain Octahedron structure for (n-levels) where $\mathrm{n}$ is even, based on degree and eccentricity of each vertex with existence of their frequencies

\begin{tabular}{c|c|c|l}
\hline $\mathbf{d}(\mathbf{u})$ & $\varepsilon(\mathbf{u})$ & frequency & Range of k and n \\
\hline 4 & $\mathrm{k}$ & 6 & $k=\frac{n+4}{2}, n \geq 4$ \\
\hline 4 & $\mathrm{k}$ & 8 & $\frac{n+6}{2} \leq k \leq n, n \geq 6$ \\
\hline 4 & $\mathrm{k}$ & 10 & $k=n+1, n \geq 4$ \\
\hline 4 & $\mathrm{k}$ & 2 & $k=n+2 n \geq 4$ \\
\hline 8 & $\mathrm{k}$ & 1 & $k=\frac{n+2}{2}, n \geq 4$ \\
\hline 8 & $\mathrm{k}$ & 2 & $\frac{n+4}{2} \leq k \leq n, n \geq 4$ \\
\hline
\end{tabular}

Table 3

Vertex partition of Chain Octahedron structure for (n-levels) where $\mathrm{n}$ is odd, based on eccentricity of each vertex with existence of their frequencies

\begin{tabular}{c|c|c}
\hline$\varepsilon(\mathbf{u})$ & frequency & Range of $\mathrm{k}$ and $\mathbf{~}$ \\
\hline $\mathrm{k}$ & 4 & $k=\frac{n+3}{2}, n \geq 3$ \\
\hline $\mathrm{k}$ & 10 & $\frac{n+5}{2} \leq k \leq n+1, n \geq 3$ \\
\hline $\mathrm{k}$ & 2 & $k=n+2, n \geq 3$ \\
\hline
\end{tabular}


Table 4

Vertex partition of Chain Octahedron structure for (n-levels) where $\mathrm{n}$ is even, based on eccentricity of each vertex with existence of their frequencies

\begin{tabular}{c|c|c}
\hline $\boldsymbol{\varepsilon ( u )}$ & frequency & Range of k and n \\
\hline $\mathrm{k}$ & 1 & $k=\frac{n+2}{2}, n \geq 2$ \\
\hline $\mathrm{k}$ & 8 & $k=\frac{n+4}{2}, n \geq 2$ \\
\hline $\mathrm{k}$ & 10 & $\frac{n+6}{2} \leq k \leq n+1, n \geq 4$ \\
\hline $\mathrm{k}$ & 2 & $k-n+2, n \geq 2$ \\
\hline
\end{tabular}

Table 5

Edge partition of Chain Octahedron structure for (n-levels) where $\mathrm{n}$ is odd, based on eccentricity of end vertices with existence of their frequencies

\begin{tabular}{c|c|c}
\hline$(\varepsilon(\mathrm{u}), \varepsilon(\mathrm{v}))$ & frequency & Range of k and n \\
\hline$(\mathrm{k}, \mathrm{k})$ & 5 & $k=\frac{n+3}{2}, n \geq 3$ \\
\hline$(\mathrm{k}, \mathrm{k}+1)$ & 14 & $k=\frac{n+3}{2}, n \geq 3$ \\
\hline$(\mathrm{k}, \mathrm{k})$ & 9 & $k=\frac{n+5}{2}, n \geq 3$ \\
\hline$(\mathrm{k}, \mathrm{k}+1)$ & 16 & $n+5$ \\
\cline { 2 - 3 } & & $\frac{2}{2}, k \leq n, n \geq 5$ \\
\hline$(\mathrm{k}, \mathrm{k})$ & 8 & 2 \\
\cline { 2 - 3 } & & $k=n+1, n \geq 3$ \\
\hline$(\mathrm{k}, \mathrm{k}+1)$ & 8 &
\end{tabular}

Table 6

Edge partition of Chain Octahedron structure for (n-levels) where $\mathrm{n}$ is even, based on eccentricity of end vertices with existence of their frequencies

\begin{tabular}{|c|c|c|}
\hline$(\varepsilon(u), \varepsilon(v))$ & frequency & Range of $k$ and $n$ \\
\hline$(\mathrm{k}, \mathrm{k}+1)$ & 8 & $k=\frac{n+2}{2}, n \geq 2$ \\
\hline$(\mathrm{k}, \mathrm{k})$ & 8 & $\frac{n+4}{2} \leq k \leq n+1, n \geq 2$ \\
\hline$(\mathrm{k}, \mathrm{k}+1)$ & 16 & $\frac{n+4}{2} \leq k \leq n, n \geq 4$ \\
\hline$(\mathrm{k}, \mathrm{k}+1)$ & 8 & $k=n+1, n \geq 2$ \\
\hline
\end{tabular}

\subsection{Eccentric Connectivity Polynomial}

Now in the following theorems, we computed the eccentric polynomial of chain Octahedron structure $\mathrm{ECP}(\mathrm{CHOn}, \mathrm{x})$.
Theorem 2.1.1. Let $\mathrm{CHOn}$, for all $\mathrm{n}>1$, where $\mathrm{n}$ is odd, be the chain Octahedron structure, then the eccentric polynomial of $\mathrm{CHOn}$ is

$$
E C P\left(\mathrm{CHO}_{n}, x\right)=8\left\{x^{\frac{n+3}{2}}+x^{n+1}(5+x)\right\}+16\left\{2 \sum_{k=\frac{n+5}{2}}^{n}(x)^{k}+\sum_{k=\frac{n+3}{2}}^{n}(x)^{k}\right\} .
$$


Proof: Let CHOn, where $\mathrm{n}$ is odd, be the chain Octahedron structure contains $5 n+1$ vertices and $12 \mathrm{n}$ edges.

The formula of eccentric polynomial is

$$
E C F(G, x)=\sum_{v \in V} d_{u} x^{\varepsilon(v)}
$$

Using the vertex partition from Table 1, we have the following computations

$$
\begin{aligned}
& E C P\left(\mathrm{CHO}_{n}, x\right)= \sum_{k=\frac{n+3}{2}} 4 \times 2 \times(x)^{k}+\sum_{k=\frac{n+5}{2}}^{n} 4 \times 8 \times(x)^{k}+\sum_{k=n+1} 4 \times 10 \times(x)^{k} \\
&+\sum_{k=n+2}^{n} 4 \times 2 \times(x)^{k}++\sum_{k=\frac{n+3}{2}}^{n} \mathrm{~s} \times 2 \times(x)^{k} \\
& \operatorname{ECP}\left(\operatorname{CS}_{n}, x\right)=8 \times(x)^{\frac{n+3}{2}}+32 \sum_{k=\frac{n+5}{2}}^{n}(x)^{k}+40 \times(x)^{n+1}+8 \times(x)^{n+2}+16 \sum_{k=\frac{n+3}{2}}^{n}(x)^{k}
\end{aligned}
$$

After an easy simplification, we get

$$
E C P\left(\text { CHO }_{n}, x\right)=8\left\{x^{\frac{n+3}{2}}+x^{n+1}(5+x)\right\}+16\left\{2 \sum_{k=\frac{n+5}{2}}^{n}(x)^{k}+\sum_{k=\frac{n+3}{2}}^{n}(x)^{k}\right\}
$$

Theorem 2.1.2. Let $\mathrm{CHOn}$, for all $\mathrm{n} \geq 2$, where the eccentric polynomial of $\mathrm{C} \mathrm{H} \mathrm{On}$ is $\mathrm{n}$ is even, be the chain Octahedron structure, then

$$
\operatorname{ECP}\left(\mathrm{CHO}_{n}, x\right)=8\left\{x^{\frac{n+3}{2}}(3 x+1)+x^{n+1}(5+x)\right\}+16\left\{2 \sum_{k=\frac{n+6}{2}}^{n}(x)^{k}+\sum_{k=\frac{n+4}{2}}^{n}(x)^{k}\right\}
$$

Proof. Let $\mathrm{CHOn}$, where $\mathrm{n}$ is even, be the chain Octahedron structure contains $5 n+1$ vertices and $12 \mathrm{n}$ edges.

The formula of eccentric polynomial is

$$
E C P(G, x)=\sum_{v \in V} d_{u} x^{z(v)}
$$

Using the vertex partition from Table 2, we have the following computations

$$
\begin{aligned}
\operatorname{ECP}\left(\mathrm{CHO}_{n}, x\right)= & \sum_{k=\frac{n+4}{2}} 4 \times 6 \times(x)^{k}+\sum_{k=\frac{n+6}{2}}^{n} 4 \times 8 \times(x)^{k}+\sum_{k=n+1} 4 \times 10 \times(x)^{k} \\
& +\sum_{k=n+2} 4 \times 2 \times(x)^{k}+\sum_{k=\frac{n+2}{2}} 8 \times 1 \times(x)^{k}+\sum_{k=\frac{n+4}{2}}^{n} 8 \times 2 \times(x)^{k} \\
E C P\left(C_{n}, x\right)= & 24 \times(x)^{\frac{n+4}{2}}+32 \sum_{k=\frac{n+6}{2}}^{n}(x)^{k}+40 \times(x)^{n+1}+8 \times(x)^{n+2}+8 \times(x)^{\frac{n+2}{2}} \\
& +16 \sum_{k=\frac{n+4}{2}}^{n}(x)^{k}
\end{aligned}
$$


After an easy simplification, we get

$$
E C P\left(\mathrm{CHO}_{n}, x\right)=8\left\{x^{\frac{n+3}{2}}(3 x+1)+x^{n+1}(5+x)\right\}+16\left\{2 \sum_{k=\frac{n+6}{2}}^{n}(x)^{k}+\sum_{k=\frac{n+4}{2}}^{n}(x)^{k}\right\}
$$

\subsection{Eccentric Connectivity Index}

Now in the following theorems, we computed the eccentric connectivity index of chain Octahedron structure $\xi(\mathrm{CHOn})$.
Theorem 2.2.1. Let CHOn, for all $n>1$, where $\mathrm{n}$ is odd, be the chain Octahedron structure, then the eccentric connectivity index of $\mathrm{CHOn}$ is

$$
\xi\left(\mathrm{CHO}_{n}\right)=4\{13 n+17\}+16\left\{2 \sum_{k=\frac{n+5}{2}}^{n} k+\sum_{k=\frac{n+3}{2}}^{n} k\right\}
$$

Proof. Let CHOn, where $\mathrm{n}$ is even, be the chain Octahedron structure contains $5 n+1$ vertices and $12 \mathrm{n}$ edges.

The formula of eccentric connectivity index is

$$
\xi(G)=\sum_{v e V} d_{v} \varepsilon(v)
$$

Using the vertex partition from Table 1, we have the following computations

$$
\begin{aligned}
& \xi\left(\mathrm{CHO}_{n}\right)=\sum_{k=\frac{n+3}{2}} 4 \times 2 \times k+\sum_{k=\frac{n+5}{2}}^{n} 4 \times 8 \times k+\sum_{k=n+1} 4 \times 10 \times k+\sum_{k=n+2} 4 \times 2 \times k \\
& +\sum_{k=\frac{n+3}{2}} 8 \times 2 \times k \\
& \quad \xi\left(C H O_{n}\right)=8 \times\left(\frac{n+3}{2}\right)+32 \sum_{k=\frac{n+5}{2}}^{n} k+40 \times(n+1)+8 \times(n+2)+16 \sum_{k=\frac{n+3}{2}}^{n} k
\end{aligned}
$$

After an easy simplification, we get

$$
\xi\left(\text { CHO }_{n}\right)=4\{13 n+17\}+16\left\{2 \sum_{k=\frac{n+5}{2}}^{n} k+\sum_{k=\frac{n+3}{2}}^{n} k\right\}
$$

Theorem 2.2.2. Let $\mathrm{CHOn}$, for all $\mathrm{n} \geq 2$, where the eccentric connectivity index of CHOn is $\mathrm{n}$ is even, be the chain Octahedron structure, then

$$
\xi\left(\mathrm{CHO}_{n}\right)=16\{4 n+7\}+16\left\{2 \sum_{k=\frac{n+6}{2}}^{n} k+\sum_{k=\frac{n+4}{2}}^{n} k\right\}
$$


Proof. Let $\mathrm{C} \mathrm{H}$ On, where $\mathrm{n}$ is even, be the chain Octahedron structure contains $5 n+1$ vertices and $12 \mathrm{n}$ edges.

The formula of eccentric connectivity index is

$$
\xi(G)=\sum_{v \in V} d_{v} \varepsilon(v)
$$

Using the vertex partition from Table 2, we have the following computations

$$
\begin{aligned}
& \xi\left(\mathrm{CHO}_{n}\right)=\sum_{k=\frac{n+4}{2}} 4 \times 6 \times k+\sum_{k=\frac{n+6}{2}}^{n} 4 \times 8 \times k+\sum_{k=n+1} 4 \times 10 \times k+\sum_{k=n+2} 4 \times 2 \times k \\
& +\sum_{k=\frac{n+3}{2}} 8 \times 2 \times k+\sum_{k=\frac{n+3}{2}}^{n} 8 \times 1 \times k \\
& \xi\left(\mathrm{CHO}_{n}\right)=24 \times\left(\frac{n+4}{2}\right)+32 \sum_{k=\frac{n+6}{2}}^{n} k+40 \times(n+1)+8 \times(n+2)+8 \times\left(\frac{n+2}{2}\right) \\
& +16 \sum_{k=\frac{n+3}{2}}^{n}
\end{aligned}
$$

After an easy simplification, we get

$$
\xi\left(\mathrm{CHO}_{n}\right)=16\{4 n+7)+16\left\{2 \sum_{k=\frac{n+6}{2}}^{n} k+\sum_{k=\frac{n+4}{2}}^{n} k\right\}
$$

\subsection{Total Eccentricity Index}

Now in the following theorems, we computed the total eccentricity index of chain Octahedron structure $\zeta$ (C H On ).

Theorem 2.3.1. Let $\mathrm{C} \mathrm{H}$ On , for all $\mathrm{n}>1$, where $\mathrm{n}$ is odd, be the chain Octahedron structure, then the total eccentricity index $\zeta$ of $\mathrm{C} \mathrm{H} \mathrm{On} \mathrm{is}$

$$
\zeta\left(\mathrm{CHO}_{n}\right)=2\{2 n+5\}+10 \sum_{k=\frac{n+5}{2}}^{n+1}(k)
$$

Proof. Let CHOn, where $\mathrm{n}$ is odd, be the chain Octahedron structure contains $5 n+1$ vertices and $12 \mathrm{n}$ edges.

The formula of total eccentricity index is

$$
\zeta(G)-\sum_{v \in \text { ic }} \varepsilon(v)
$$

Using the vertex partitioned from Table 3, we have the following computations

$$
\zeta\left(\text { CHO }_{n}\right)=4 \sum_{k=\frac{n+3}{2}}(k)+10 \sum_{k=\frac{n+5}{2}}^{n+1}(k)+2 \sum_{k=n+2}(k)
$$

$$
\zeta\left(\mathrm{CHO}_{n}\right)=4\left(\frac{n+3}{2}\right)+10 \sum_{k=\frac{n+5}{2}}^{n+1}(k)+2(n+2)
$$

After an easy simplification, we get

$$
\zeta\left(\mathrm{CHO}_{n}\right)=2\{2 n+5\}+10 \sum_{k=\frac{n+5}{2}}^{n+1}(k)
$$

Theorem 2.3.2. Let $\mathrm{CH}$ On, for all $\mathrm{n} \geq 2$, where $\mathrm{n}$ is even, be the chain Octahedron structure, then the total eccentricity index $\zeta$ of $\mathrm{C} \mathrm{H}$ On is 


$$
\zeta\left(C H O_{n}\right)=\frac{1}{2}\{13 n+42\}+10 \sum_{k=\frac{n+6}{2}}^{n+1}(k)
$$

Proof. Let $\mathrm{C} \mathrm{H}$ On, where $\mathrm{n}$ is even, be the chain Octahedron structure contains $5 n+1$ vertices and $12 n$ edges.

The formula of total eccentricity index is

$$
\begin{gathered}
\zeta\left(\mathrm{CHO}_{n}\right)=1 \sum_{k=\frac{n+2}{2}}(k)+8 \sum_{k=\frac{n+4}{2}}(k)+10 \sum_{k=\frac{n+6}{2}}^{n+1}(k)+2 \sum_{k=n+2}(k) \\
\zeta\left(\mathrm{CHO}_{n}\right)=\frac{n+2}{2}+8\left(\frac{n+4}{2}\right)+10 \sum_{k=\frac{n+6}{2}}^{n+1}(k)+2(n+2)
\end{gathered}
$$

Using the vertex partitioned from Table 4, we have the following computations

After an easy simplification, we get

$$
\zeta\left(\mathrm{CHO}_{n}\right)=2[2 n+5\}+10 \sum_{k=\frac{n+5}{2}}^{n+1}(k) .
$$

\subsection{Average Eccentricity Index}

In this section we find the average eccentricity index of chain Octahedron structure avec(C H On).

Theorem 2.4.1. Let $\mathrm{C} H$ On, for all $\mathrm{n}>1$, where $\mathrm{n}$ is odd, be the chain Octahedron structure, then the average eccentricity index avec( $\mathrm{C} \mathrm{H} \mathrm{On})$ is

$$
\begin{aligned}
& \operatorname{avec}\left(\mathrm{CHO}_{n}\right)=\frac{2}{5 n+1}\left[2 n+5+5 \sum_{k=\frac{n+5}{2}}^{n+1}(k)\right] \\
& \operatorname{avec}\left(\mathrm{CHO}_{n}\right)=\frac{1}{5 n+1}\left\{4 \sum_{k=\frac{n+3}{2}}(k)+10 \sum_{k=\frac{n+5}{2}}^{n+1}(k)+2 \sum_{k=n+2}^{n}(k)\right\} \\
& \operatorname{avec}\left(\mathrm{CHO}_{n}\right)=\frac{1}{5 n+1}\left\{4\left(\frac{n+3}{2}\right)+10 \sum_{k=\frac{n+5}{2}}^{n+1}(k)+2(n+2)\right\}
\end{aligned}
$$

After an easy simplification, we get

$$
\operatorname{avec}\left(\mathrm{CHO}_{n}\right)=\frac{2}{5 n+1}\left\{2 n+5+5 \sum_{k=\frac{n+5}{2}}^{n+1}(k)\right\}
$$


Theorem 2.4.2. Let $\mathrm{C} \mathrm{H}$ On , for all $n \geq 2$, where $\mathrm{n}$ is even, be the chain Octahedron structure, then the average eccentricity index avec $(\mathrm{C}$ H On ) is

$$
\operatorname{avec}\left(\mathrm{CHO}_{n}\right)=\frac{1}{2(5 n+1)}\left\{13 n+42+20 \sum_{k=\frac{n+6}{2}}^{n+1}(k)\right\}
$$

Proof. Let $\mathrm{C} \mathrm{H}$ On, where $\mathrm{n}$ is even, be the chain Octahedron structure contains $5 n+1$ vertices and $12 \mathrm{n}$ edges.

The formula of average eccentricity index is

$$
\operatorname{avec}(G)=\frac{1}{n} \sum s_{i}
$$

Using the vertex partitioned from Table 4, we have the following computations

$$
\operatorname{avec}\left(\text { CHO }_{n}\right)=\frac{1}{5 n+1}\left\{1 \sum_{k=\frac{n+2}{2}}(k)+8 \sum_{k=\frac{n+4}{2}}(k)+10 \sum_{k=\frac{n+6}{2}}^{n+1}(k)+2 \sum_{k=n+2}(k)\right\}
$$

avec $\left(\right.$ CHO $\left._{n}\right)=\frac{1}{5 n+1}\left\{\frac{n+2}{2}+8\left(\frac{n+4}{2}\right)+10 \sum_{k=\frac{n+6}{2}}^{n+1}(k)+2(n+2)\right\}$

After an easy simplification, we get

$$
\operatorname{avec}\left(\mathrm{CHO}_{n}\right)=\frac{1}{2(5 n+1)}\left\{13 n+42+20 \sum_{k=\frac{n+6}{2}}^{n+1}(k)\right\}
$$

\subsection{Geometric-arithmetic Index}

In this section we find the eccentricity based geometric-arithmetic index of chain Octahedron structure GA4 (C H On ).
Theorem 2.5.1. Let $\mathrm{C} H$ On for all $\mathrm{n}>1$, where $\mathrm{n}$ is odd, be the chain Octahedron structure, then the geometric-arithmetic index GA4 ( $\mathrm{C} \mathrm{H} \mathrm{On} \mathrm{)} \mathrm{is}$

$$
G A_{4}\left(C H O_{n}\right)=2\left\{2 n+1+\frac{7 \sqrt{n^{2}+8 n+15}}{n+4}+\frac{8 \sqrt{n^{2}+3 n+2}}{2 n+3}\right\}+32 \sum_{k=\frac{n+5}{2}}^{n} \frac{\sqrt{k(k+1)}}{2 k+1}
$$

Proof. Let $\mathrm{C} \mathrm{H}$ On , where $\mathrm{n}$ is odd, be the chain Octahedron structure contains $5 n+1$ vertices and $12 \mathrm{n}$ edges.

The general formula of eccentricity based geometric arithmetic index is

$$
G A_{4}=\sum_{w v E(G)} \frac{2 \sqrt{\varepsilon(u) \times \varepsilon(v)}}{\varepsilon(u)+\varepsilon(v)}
$$

Using the edge partitioned from Table 5, we have the following computations

$$
G A_{4}\left(\mathrm{CHO}_{n}\right)=5 \sum_{k=\frac{n+3}{2}} \frac{2 \sqrt{k \times k}}{k+k}+14 \sum_{k=\frac{n+3}{2}} \frac{2 \sqrt{k \times(k+1)}}{k+k+1}+9 \sum_{k=\frac{n+5}{2}} \frac{2 \sqrt{k \times k}}{k+k}
$$




$$
\begin{aligned}
&+16 \sum_{k=\frac{n+5}{2}}^{n} \frac{2 \sqrt{k \times(k+1)}}{k+k+1}+8 \sum_{k=\frac{n+7}{2}}^{n+1} \frac{2 \sqrt{k \times k}}{k+k}+8 \sum_{k=n+1} \frac{2 \sqrt{k \times(k+1)}}{k+k+1} \\
& \mathrm{xA}_{4}\left(\mathrm{CHO} \mathrm{O}_{n}\right)= 10 \sum_{k=\frac{n+3}{2}} \frac{\sqrt{k^{2}}}{2 k}+28 \sum_{k=\frac{n+3}{2}} \frac{\sqrt{k \times(k+1)}}{2 k+1}+18 \sum_{k+\frac{n+5}{2}} \frac{\sqrt{k^{2}}}{2 k} \\
&+32 \sum_{k=\frac{n+5}{2}} \frac{\sqrt{k \times(k+1)}}{2 k+1}+16 \sum_{k=\frac{n+7}{2}} \frac{\sqrt{k^{2}}}{2 k}+16 \sum_{k=n+1} \frac{\sqrt{k \times(k+1)}}{2 k+1}
\end{aligned}
$$

After an easy simplification, we get

$$
G A_{4}\left(\mathrm{CHO}_{n}\right)=2\left\{2 n+1+\frac{7 \sqrt{n^{2}+8 n+15}}{n+4}+\frac{8 \sqrt{n^{2}+3 n+2}}{2 n+3}\right\}+32 \sum_{k=\frac{n+5}{2}}^{n} \frac{\sqrt{k(k+1)}}{2 k+\mathbf{1}}
$$

Theorem 2.5.2. Let C H On for all $n \geq 2$, where the geometric-arithmetic index GA4 (C H On ) is $\mathrm{n}$ is even, be the chain Octahedron structure, then

$$
G A_{4}\left(\mathrm{CHO}_{n}\right)=8\left\{\frac{n}{2}+\frac{\sqrt{n^{2}+G n+0}}{n+3}+\frac{2 \sqrt{n^{2}+3 n+2}}{2 n+3}\right\}+3: 2 \sum_{k=\frac{n+4}{2}}^{n} \frac{\sqrt{k(k+1)}}{2 k+1}
$$

Proof. Let C H On, where $\mathrm{n}$ is even, be the chain Octahedron structure contains $5 n+1$ vertices and $12 \mathrm{n}$ edges.

The general formula of eccentricity based geometric arithmetic index is

$$
G A_{4}=\sum_{u v \in E(C)} \frac{2 \sqrt{\varepsilon(u) \times \varepsilon(v)}}{\varepsilon(u)+\varepsilon(v)}
$$

Using the edge partitioned from Table 6, we have the following computations

$$
\begin{aligned}
G A_{4}\left(\mathrm{CHO}_{n}\right)= & 8 \sum_{k=\frac{n+2}{2}} \frac{2 \sqrt{k \times(k+1)}}{k+k+1}+8 \sum_{k=\frac{n+4}{2}}^{n+1} \frac{2 \sqrt{k \times k}}{k+k}+16 \sum_{k=\frac{n+4}{2}}^{n} \frac{2 \sqrt{k \times(k+1)}}{k+k+1} \\
& +8 \sum_{k=n+1} \frac{2 \sqrt{k \times(k+1)}}{k+k+1} \\
G A_{4}\left(\mathrm{CHO}_{n}\right)= & 16 \sum_{k=\frac{n+2}{2}} \frac{\sqrt{k \times(k+1)}}{2 k+1}+16 \sum_{k=\frac{n+4}{2}}^{n} \frac{\sqrt{k^{2}}}{2 k}+32 \sum_{k=\frac{n+4}{2}}^{n} \frac{\sqrt{k \times(k+1)}}{2 k+1} \\
& +16 \sum_{k=n+1} \frac{\sqrt{k \times(k+1)}}{2 k+1}
\end{aligned}
$$

After an easy simplification, we get 


$$
G A_{4}\left(\mathrm{CHO}_{n}\right)=8\left\{\frac{n}{2}+\frac{\sqrt{n^{2}+6 n+0}}{n+3}+\frac{2 \sqrt{n^{2}+3 n+2}}{2 n+3}\right\}+32 \sum_{k=\frac{n+4}{2}}^{n} \frac{\sqrt{k(k+1)}}{2 k+1}
$$

\subsection{Atom bond Connectivity Index}

In this section we find the eccentricity based atom bond connectivity index of chain Octahedron structure ABC5 (C H On).
Theorem 2.6.2. Let $\mathrm{C} H$ On for all $n \geq 2$, where $\mathrm{n}$ is odd, be the chain Octahedron structure, then the atom bond connectivity index ABC5 (C H On) is

$$
\begin{aligned}
A B C_{5}\left(\mathrm{CHO}_{n}\right) & =2\left\{\frac{5 \sqrt{n+1}}{n+3}+\frac{9 \sqrt{n+3}}{n+5}+\frac{4 \sqrt{2 n}}{n+1}\right\}+4\left\{2 \sqrt{\frac{2 n+1}{n^{2}+3 n+2}}+8 \sqrt{\frac{n+4}{n^{2}+12 n+35}}\right. \\
& +7 \sqrt{\frac{n+2}{n^{2}+8 n+15}+8 \sum_{k=\frac{n+7}{2}}^{n}\left\{\sqrt{\frac{2 k-1}{k(k+1)}+\frac{\sqrt{2 k-2}}{k}}\right\}}
\end{aligned}
$$

Proof. Let $\mathrm{C} \mathrm{H}$ On, where $\mathrm{n}$ is odd, be the chain Octahedron structure contains $5 n+1$ vertices and $12 \mathrm{n}$ edges.

The general formula of eccentricity based atom bond connectivity index is

$$
A B C_{\mathrm{E}}=\sum_{\text {w }} \sqrt{\frac{\varepsilon(u)+\varepsilon(G)+2}{\varepsilon(u) \times \varepsilon(v)}}
$$

Using the edge partitioned from Table 5, we have the following computations

$$
\begin{aligned}
& A B C_{5}\left(\mathrm{CHO}_{n}\right)=5 \sum_{k=\frac{n+3}{2}} \sqrt{\frac{k+k-2}{k \times k}}+5 \sum_{k=\frac{n+3}{2}} \sqrt{\frac{k+k-1}{k \times(k+1)}}+9 \sum_{k=\frac{n+5}{2}} \sqrt{\frac{k+k-2}{k \times k}} \\
& +16 \sum_{k=\frac{n+5}{2}}^{n} \sqrt{\frac{k+k-1}{k \times(k+1)}}+8 \sum_{k=\frac{n+7}{2}}^{n+1} \sqrt{\frac{k+k-2}{k \times k}}+8 \sum_{k=n+1} \sqrt{\frac{k+k-1}{k \times(k+1)}}
\end{aligned}
$$

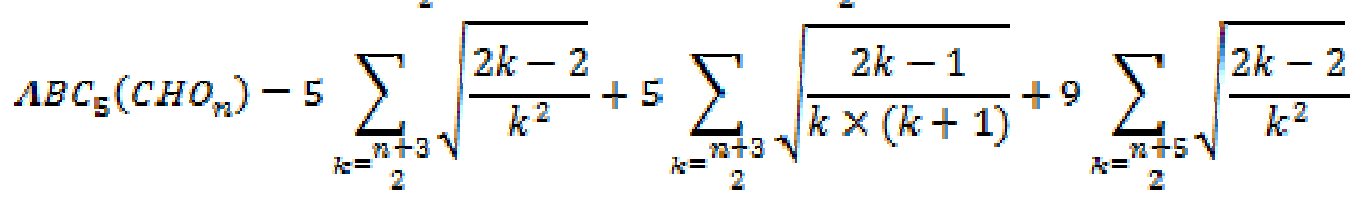

$$
\begin{aligned}
& +16 \sum_{k=\frac{n+5}{2}}^{n} \sqrt{\frac{2 k-1}{k \times(k+1)}}+8 \sum_{k=\frac{n+7}{2}}^{n+1} \sqrt{\frac{2 k-2}{k^{2}}}+8 \sum_{k=n+1} \sqrt{\frac{2 k-1}{k \times(k+1)}}
\end{aligned}
$$

After some simplification, we obtain

$$
\begin{aligned}
A B C_{5}\left(C H O_{n}\right) & =2\left\{\frac{5 \sqrt{n+1}}{n+3}+\frac{9 \sqrt{n+3}}{n+5}+\frac{4 \sqrt{2 n}}{n+1}\right\}+4\left\{2 \sqrt{\frac{2 n+1}{n^{2}+3 n+2}}+8 \sqrt{\frac{n+4}{n^{2}+12 n+35}}\right. \\
& +7 \sqrt{\frac{n+2}{n^{2}+8 n+15}+8 \sum_{k=\frac{n+7}{2}}^{n}\left\{\sqrt{\frac{2 k-1}{k(k+1)}+\frac{\sqrt{2 k-2}}{k}}\right\}}
\end{aligned}
$$


Theorem 2.6.2. Let $\mathrm{C}$ H On for all $\mathrm{n} \geq 2$, where $\mathrm{n}$ is even, be the chain Octahedron structure, then the atom bond connectivity index $\mathrm{ABC} 5$ ( $\mathrm{C} \mathrm{H} \mathrm{On} \mathrm{)}$ is

$$
\begin{aligned}
A B C_{5}\left(C H O_{n}\right) & =8\left[2 \sqrt{\frac{n+1}{n^{2}+6 n+8}}+\sqrt{\frac{2 n+1}{n^{2}+3 n+2}}\right]+8\left[\sum_{k=\frac{n+4}{2}}^{n+1} \frac{\sqrt{2(k-1)}}{k}\right. \\
& \left.+2 \sum_{k=\frac{n+4}{2}}^{n} \frac{\sqrt{2(k-1)}}{k(k+1)}\right\}
\end{aligned}
$$

Proof. Let C H On, where $\mathrm{n}$ is even, be the chain Octahedron structure contains $5 n+1$ vertices and $12 \mathrm{n}$ edges.

The general formula of eccentricity based atom bond connectivity index is

$$
A B C_{\mathrm{E}}=\sum_{\text {w } E_{(G)}} \sqrt{\frac{\varepsilon(u)+\varepsilon(v)-2}{\varepsilon(u) \times \varepsilon(v)}}
$$

Using the edge partitioned from Table 6, we have the following computations

$$
\begin{aligned}
& A B C_{5}\left(\mathrm{CHO}_{n}\right)=8 \sum_{k=\frac{n+2}{2}} \sqrt{\frac{k+k-1}{k \times(k+1)}}+8 \sum_{k=\frac{n+4}{2}}^{n+1} \sqrt{\frac{k+k-2}{k \times k}}+16 \sum_{k=\frac{n+4}{2}}^{n} \sqrt{\frac{2 k-1}{k \times(k+1)}} \\
& +8 \sum_{k=n+1} \sqrt{\frac{k+k-1}{k \times(k+1)}} \\
& A B C_{\mathrm{s}}\left(\mathrm{CHO}_{n}\right)=8 \sum_{k=\frac{n+2}{2}} \sqrt{\frac{2 k-1}{k \times(k+1)}}+8 \sum_{k=\frac{n+4}{2}}^{n+1} \sqrt{\frac{2 k-2}{k^{2}}}+16 \sum_{k=\frac{n+4}{2}}^{n} \sqrt{\frac{2 k-1}{k \times(k+1)}} \\
& +8 \sum_{k=n+1} \sqrt{\frac{2 k-1}{k \times(k+1)}}
\end{aligned}
$$

After some simplification, we obtain

$$
\begin{aligned}
A B C_{5}\left(C H O_{n}\right) & =8\left\{2 \sqrt{\frac{n+1}{n^{2}+6 n+8}}+\sqrt{\frac{2 n+1}{n^{2}+3 n+2}}\right\}+8\left\{\sum_{k=\frac{n+4}{2}}^{n+1} \frac{\sqrt{2(k-1)}}{k}\right. \\
& \left.+2 \sum_{k=\frac{n+4}{2}}^{n} \frac{\sqrt{2(k-1)}}{k(k+1)}\right\}
\end{aligned}
$$

\section{CONCLUSIONS}

In this paper, we computed the eccentric connectivity polynomial EC P (C H On , x), eccentric connectivity index $\xi(\mathrm{C} \mathrm{H}$ On $)$, total eccentricity index $\zeta(\mathrm{C} \mathrm{H}$ On $)$, average eccentricity index avec(C $\mathrm{H}$ On $)$, Atom bond connectivity index ABC5 (C H On ) and Geometric arithmetic index GA4 (C H On ) of Chain Octahedron Structure.

\section{REFERENCES}

1. M. Alaeiyan and J. Asadpour, Optoelectron. Adv. Mater. Rapid Commun., 2012, 6, 191-193.

2. M. Alaeiyan, R. Mojarad and J. Asadpour, Optoelectron. Adv. Mater. Rapid Commun., 2011, 5, 761-763.

3. P. Dankelmann, W. Goddard and C. S. Swart, Utilitas Math., 2004, 65, 41 - 51 .

4. Z. Ahmad, M. Naseem, M. K. Jamil, M. K. Siddiqui and M. F. Nadeem, Eurasian Chem. Commun., 2020, 2, 663-671. 
5. W. Gao, M. R. Farahani and M. K. Jamil, Acta Chim. Slov., 2016, 63, 376-379.

6. W. Gao, W. Wang, M. K. Jamil and M. R. Farahani, J. Chem., 2016, 7, 88-98.

7. W. Gao, W. F. Wang, M. K. Jamil, R. Farooq and M. R. Farahani, Bulgarian Chem. Comm., 2016, 48, 543 - 549.

8. M. Ghorbani and A. Khaki, Optoelectron. Adv. Mater. Rapid Comm., 2010, 4, $2212-2215$.

9. M. Ghorbani, and M. A. Hosseinzadeh, Filomat, 2012, $26,93-100$.

10. S. Hayat and M. Imran, Appl. Mathem. Computation., 2014, 240, 213 - 228.

11. S. Hayat, M. A. Malik and M. Imran, Romanian J. Info. Scie. Techn., 2015, 18, $144-165$.

12. S. Hayat and M. Imran, J. Comput. Theor. Nanoscie., 2015, 12, $533-541$.

13. A. Ilic, Comput. Math. Appl., 2012, 64, $2877-2885$.

14. M. Imran and S. Hayat, Sci. Int.(Lahore), 2014, 26, 1407 1412.

15. J. J. Perry Iv, J. A. Perman and M. J. Zaworotko, Chem. Soc. Rev., 2009, 38, $1400-1417$.

16. A. M. Hinz and D. Parisse, Graphs and Combinatorics, 2012, $5,671-686$.

17. A. Q. Baig, M. Imran and H. Ali, Canadian J. Chem., 2015, 93, $730-739$.

18. A. Q. Baig, M. R. Azhar, M. R. Farahani and S. Ediz, Comm. Appl. Anal., 2017, 21, $631-646$.

19. M. R. Farahani, Acta Chim. Slov., 2013, 60, $429-432$.

20. M. R. Farahani, Appl. Scie. J. Chem., 2013, 21,1260-1265.

21. B. Rajan, A. William, C. Grigorious and S. Stephen, J. Comp. Math. Sci., 2012, 3, 530 - 535.
22. V. Sharma, R. Goswami and A. K. Madan, J. Chem. Inf. Comput. Sci., 1997, 37, 273-282.

23. Y. Tang and B. Zhou, MATCH Commun Math. Comput. Chem., 2012, 67, 405-423.

24. B. Basavanagoud, W. Gao, S. Patil, V. R. Desai, K. G. Mirajkar, and B. Pooja, Appl. Mathem. Nonlinear Scie., 2017,2, 285-298.

25. D. Liu, C. Wang and S. Wang, Appl. Mathem. Nonlinear Scie., 2018, 3, 419-426, 2018.

26. J. A. Aledo, L. G. Diaz, S. Martinez and J. C. Valverde, Appl. Mathem. Nonlinear Scie., 2018, 3, 593-602.

27. M. Knor, R. Skrekovski and A. Tepeh, Appl. Mathem. Nonlinear Scie., 2018, 3, 4334-46.

28. J.-B. Liu, C. Wang, S. Wang and B. Wei, Bull. Malaysian Mathem. Scie. Soc., 2019, 42, 67-78.

29. J.-B. Liu, J. Zhao and Z. X. Zhu, Int. J. Quantum Chem., 2019, 119, 59-71.

30. J.-B. Liu, J. Zhao, J. Min and J. D. Cao, FractalsComplex Geometry Patterns and Scaling in Nature and Society, 2019, 27, 195-215.

31. J.-B. Liu, J. Zhao and Z. Cai, Physica A, 2020, 540, $123-$ 133.

32. J.-B. Liu, J. Zhao, H. He and Z. Shao, J. Statistical Phys., 2019, 177, 1131-1147.

33. M. K. Siddiqui, M. Imran and M. Saeed, Hacet. J. Math. Stat., 2020, 49, 195 - 207.

34. D. Vukic 'evic' and A. Graovac, Acta Chim. Slov., 2010, $57,524-528$.

35. M. Y. Li and S. C. Sevov, Cryst. Eng. Comm., 2013, 15, $5107-5113$. 
